\title{
Importancia de los índices periodontales en la evaluación de los implantes osteointegrados
}

\author{
ZORRILLA ROMERA C * \\ VALLECILLO CAPILLA M * *
}

\begin{abstract}
Zorrilla Romera C,Vallecillo Capilla M. Importancia de los índices periodontales en la evolución de los implantes osteointegrados. Av Periodon Implantol. 2002; 14, 2: 75-79.
\end{abstract}

\begin{abstract}
RESUMEN
El objetivo del estudio es conocer la relación existente entre la tasa de fracaso de una muestra de implantes recubiertos de hidroxiapatita (H.A.) y los parámetros periodontales del paciente; así como el grado de asociación entre estos últimos.
\end{abstract}

La muestra está formada por 52 pacientes, a los que hemos colocado 158 implantes recubiertos de H.A. (Integral ${ }^{\circledR}$ de Calcitek), que han sido revisados a los tres años de su colocación. El fracaso de los implantes se ha establecido según los criterios de SCHNITMANY SHULMAM de 1979.

El estudio realizado es observacional analítico retrospectivo de seguimiento, a través del cual se evalúa la evolución clínica y radiológica de los implantes; resultando que la recesión periimplante medida en vestibular, lingual, mesial y distal de cada fijación es el único parámetro periodontal asociado al fracaso del implante $(\mathrm{P}=0.008)$. La reabsorción ósea y la recesión periimplante se asocian de forma significativa $(\mathrm{P}<0.05)$; así como la profundidad de sondaje lo hace con el índice de gingivitis en todas sus posiciones, y con el índice de placa en posición lingual.

Hemos hallado una correlación positiva entre estos dos índices $\mathrm{r}=0.519, \mathrm{P}<0.01$ de forma que al aumentar uno lo hace el otro y viceversa. Podemos concluir que la aparición de recesión alrededor de un implante aumenta las probabilidades de que éste fracase. Existe además una correlación positiva entre el índice de gingivitis, de placa y la profundidad de bolsa, lo que indica la salud del surco gingival pero no las probabilidades de éxito del implante.

\section{PALABRAS CLAVE}

Implante de hidroxiapatita. Parámetros periodontales periimplantarios. Oseointegración. Implante oseintegrado. Tejido periimplantario. Periimplantitis.

\section{INTRODUCCIÓN}

La interfase tejido conectivo y superficie del implante difiere de la que se produce entre este tejido y el diente natural, debido a la ausencia de ligamento periodontal que separe el implante del hueso. Para autores como Ericsson y Lindhe ${ }^{1}$, así como Brägger ${ }^{2}$,
Becker $^{3}$, y Nishimura ${ }^{4}$, estas diferencias estructurales se traducen en una mayor fragilidad del epitelio de unión alrededor de los implantes en relación a la dentición natural.

En el presente estudio hemos utilizado índices perio-

* C/ Compás de San Jerónimo N 2, 5 C. 18002 Granada. Teléfono, 958 293730, Fax 958255364.

** Profesor titular de Cirugía Bucal y Fundamentos de Cirugía Maxilofacial de la Facultad de Odontología de la Universidad de Granada. 
dontales para valorar la situación clínica de los tejidos periimplantarios, al igual que hicieran autores como Lekholm ${ }^{5}$, Adell ${ }^{6}$ y Cox ${ }^{7}$, cuyos estudios, sin embargo, no revelan especificidad de estos índices para los tejidos blandos que rodean al implante, basados en estudios de seguimiento a largo plazo.

El objetivo de este trabajo es conocer, por un lado, la relación que existe entre los parámetros periodontales y el fracaso o éxito de los implantes; y por otro, la asociación que se establece entre todos los índices periodontales utilizados.

\section{MATERIAL Y MÉTODO}

La muestra objeto de estudio está formada por 52 pacientes (27 mujeres y 25 hombres) a los que se les ha colocado un total de 158 implantes recubiertos de H.A. ( Integral ${ }^{\circ}$ ); la revisión de éstos se ha llevado a cabo a los 3 años tras su colocación.

El tipo de estudio realizado es observacional analítico retrospectivo de seguimiento, a través del cual hemos seguido la evolución clínica y radiológica de los implantes mediante:

\section{A.- ÍNDICE DE PLACA DE SILNESS Y LÖE ${ }^{8}$}

Estos autores consideran el grosor de la placa a lo largo del borde gingival ya que es la que desempeña un papel en la gingivitis.

- Grado 0. Ninguna placa.

- Grado 1. Película fina de placa en el borde gingival, sólo reconocible por frotis con la sonda.

- Grado 2. Moderada placa a lo largo del borde gingival; espacios interdentarios libres; reconocible a simple vista.

- Grado 3. Mucha placa a lo largo del borde gingival; espacios interdentales ocupados por placa.

\section{B.- ÍNDICE DE GINGIVITIS DE LÖEY SILNESS ${ }^{9}$}

Este índice se basa en la concepción de la hemorragia como un criterio importante de inflamación; para determinarlo se aplica una sonda periodontal de punta redonda (sonda de la OMS) en el surco gingival. Existen 4 grados:

- Grado 0. Encía normal, ninguna inflamación, ningún cambio de color, ninguna hemorragia.

- Grado 1. Inflamación leve, ligero cambio de color, pequeña alteración de la superficie, ninguna hemorragia.
- Grado 2. Inflamación moderada, enrojecimiento, hinchazón, hemorragia al sondar y a la presión.

- Grado 3. Fuerte inflamación, enrojecimiento intenso, hinchazón, tendencia a las hemorragias, eventualmente ulceración.

\section{C.- GRADO DE MOVILIDAD DE MÏLLER ${ }^{10}$}

La causa más importante de movilidad es la pérdida cuantitativa de tejido de soporte, debida a la periimplantitis.

Para determinar el grado de movilidad, se apoyan dos mangos de espejo sobre el diente y se aplican fuerzas en sentido bucolingual: se evalúan la movilidad vestibular, lingual, mesial, y distal. El grado de movilidad se establece en base a una percepción subjetiva del observador:

- Grado 0. No hay movimiento.

- Grado 1. Aumento apreciable de la movilidad.

- Grado 2. Movilidad visible pero < de $1 \mathrm{~mm}$.

- Grado 3. Movilidad mayor de $1 \mathrm{~mm}$ en cualquier dirección.

\section{D.- PROFUNDIDAD DE SONDAJEY PÉRDIDA DE INSERCIÓN}

Los síntomas principales de una periimplantitis son: destrucción del tejido de soporte periimplantario, pérdida de inserción y la formación de bolsas en la encía. Por ello forma parte de un reconocimiento básico la medida de la profundidad de bolsa y la pérdida de inserción, para lo cual utilizamos una sonda periodontal de la OMS; así la profundidad de bolsa es la distancia entre el fondo del surco gingival y el margen de la encía; y la pérdida de inserción la distancia entre el fondo del surco gingival y el límite amelocementario, la medición la realizamos en cada uno de los cuatro cuadrantes que rodean al implante, mesial, distal, lingual y vestibular. ${ }^{11}$

\section{E.- RECESIÓN GINGIVAL}

Se mide en milímetros por V, L, D, y mesial de cada implante con una sonda periodontal de la OMS, es la distancia desde el límite amelocementario ${ }^{12}$ al margen libre de la encía.

\section{F.- PÉRDIDA DE INSERCIÓN}

Es la suma en milímetros de la recesión y la profundidad de bolsa. 


\section{G.- PÉRDIDA ÓSEA PERIIMPLANTARIA}

La reabsorción periimplantaria se determina midiendo sobre radiografías periapicales tomadas con la técnica del paralelismo, los milímetros de hueso perdido utilizando el método de la lente de aumento de Zeiss, Flubacher \& CO, Horchen-Zch ${ }^{13}$.

El fracaso del implante lo hemos establecido basándonos en los criterios de éxito de Schnitman y Shulman de $1979^{14}$, según éstos consideramos que un implante fracasa cuando:

1. Está ausente de la cavidad oral.

2. Permaneciendo en boca presenta una sola de las situaciones clínicas siguientes:

- Movilidad mayor de $1 \mathrm{~mm}$ en cualquier dirección.

- Radiolucidez periimplantaria generalizada.

- Pérdida ósea mayor de un tercio de la altura del implante.

- Sintomas persistentes y/o irreversibles como, dolor, parestesias y disestesias, infección persistente.

- Violación de estructuras nobles: seno maxilar, suelo de fosas nasales o conducto mandibular, siempre que exista sintomatología.

- Lesión en dientes vecinos, si, como consecuencia de ello, tiene que ser extraído el diente o el implante; si no es así, se considera complicación.

\section{RESULTADOS}

El porcentaje de éxito obtenido en el estudio a los 3 años de la colocación de los implantes es de un $92.4 \%$.

\section{Parámetros periodontales asociados al fracaso de los implantes}

De todos los parámetros periodontales medidos durante la revisión a los dos años tras la conexión de la prótesis, el único asociado al fracaso del implante ha sido la Recesión Periimplante Global ô=8.5. Esta recesión es la suma de las recesiones medidas en mesial, distal, vestibular y lingual

A cada implante. Al estudiar esta variable en el análisis multivariante fue dicotomizada es decir cortada en una recesión periimplantaria global mayor o igual a 2 $\mathrm{mm}$ y menor de $2 \mathrm{~mm}$; pudiendo concluir de dicho análisis que los implantes en los que la recesión global es superior o igual a $2 \mathrm{~mm}$, la probabilidad de que fracasen es de 8.5 veces superior a aquellos con recesión menor a $2 \mathrm{~mm}$.

\section{Asociación entre los distintos parámetros periodontales entre sí}

Una vez realizado el análisis bivariante entre los distintos parámetros periodontales medidos en el momento de la revisión, hemos obtenido varias asociaciones:

a.- Existe asociación significativa $(\mathrm{P}<0.05)$ entre la reabsorción ósea periimplantaria mesial y distal, y todas las recesiones mesial, distal, vestibular y lingual; esto quiere decir que cuanto mayor sea la reabsorción, mayor es la recesión y viceversa. Tabla 1.

\begin{tabular}{|l|c|c|c|c|}
\hline Correlación & R. vestibular & Lingual & Mesial & Distal \\
\hline MESIAL & $.209(*)$ & $.247(* *)$ & $.211(*)$ & $.269(* *)$ \\
\hline DISTAL & $.241(* *)$ & $.254(* *)$ & $.259(* *)$ & $.302(* *)$ \\
\hline
\end{tabular}

Tabla 1: Correlaciones entre reabsorciones y recesiones.

* El coeficiente de correlación es significativo al $5 \%$.

** El coeficiente de correlación es significativo al $1 \%$.

b.- En nuestro estudio la profundidad de sondaje está asociada al índice de gingivitis en todas sus posiciones y al índice de Placa en la posición lingual.

c.- Por otro lado existe una correlación positiva entre estos dos índices, de forma que al aumentar uno, lo hace el otro y viceversa, $r=0.519, \mathrm{P}<0.001$. Tabla 2 .

\begin{tabular}{|l|c|c|}
\hline & I. PLACA & I. GINGIVITIS \\
\hline S. VESTIBULAR $\mathrm{r}$ & $.293^{* *}$ & $.000(\mathrm{NS})$ \\
\hline S. LINGUAL $\mathrm{r}$ & $.281^{* *}$ & $.237^{*}$ \\
\hline S. MESIAL $\mathrm{r}$ & $.271^{* *}$ & $.166(\mathrm{NS})$ \\
\hline S. DISTAL $\mathrm{r}$ & $.310^{* *}$ & $.114(\mathrm{NS})$ \\
\hline
\end{tabular}

Tabla 2: Correlaciones entre la profundidad de sondaje periimplante $y$ el indice de placa y gingivitis del implante.

\section{DISCUSIÓN}

La asociación entre la recesión periimplantaria y el fracaso del implante la pusieron de manifiesto Brägger y cols ${ }^{2}$ al considerar que la existencia de dicha recesión es un factor causal de la pérdida de la oseointegración, y por tanto lleva al fracaso del tratamiento implantológico, ya que si el sellado biológico alrededor de la fijación se rompe o no existe se forma una bolsa periodontal quedando la zona expuesta a la progresión de la enfermedad periodontal. Ten Cate y cols ${ }^{15}$ opinan que el tejido conectivo gingival es el principal protector ante el medio externo de la interfase hueso implante; este tejido defiende la zona críti- 
ca de osteointegración de aquellas agresiones que provengan de dicho medio, de ahí la importancia de los parámetros clínicos periodontales

En nuestro estudio si bien hemos encontrado una asociación significativa entre la aparición de recesión y la pérdida ósea, no hemos podido determinar si la aparición de recesión periimplantaria condiciona la pérdida ósea o, por el contrario, la pérdida de hueso alrededor de la fijación se ve acompañada de una migración hacia apical del epitelio de unión.

Algunos autores como Misch ${ }^{16}$ y Lekholm ${ }^{5}$ han demostrado, al igual que nosotros, una correlación entre la profundidad de bolsa el índice de placa y de gingivítis, no encontrando asociación de estos parámetros con la pérdida de hueso. En la misma línea Lindhe y cols ${ }^{17}$ llevaron a cabo un estudio con enfermos periodontales y observaron que la progresión de la enfermedad periodontal no tiene relación con la profundidad del surco crevicular. Otros estudios como los de Becker y cols ${ }^{3}$ están en claro desacuerdo con los anteriores al encontrar asociación de la pérdida de hueso al rededor de los implantes y un aumento de la profundidad de bolsa, para Nishimura y cols ${ }^{18}$ la profundidad de sondaje es un parámetro fundamental para determinar el éxito o fracaso del implante. Para Mc Kinney ${ }^{19}$ Ericsson ${ }^{20}$, Bert ${ }^{21}$, Leonhart ${ }^{22}$ y Steflik ${ }^{23}$ y Ten Cate ${ }^{15}$ el implante dental es una unidad débil sin inserción de tejido conjuntivo ni de células epiteliales, por lo que cualquier alteración gingival secundaria a una mala higiene bucal constituye un desastre y hace fracasar el sistema.

Al igual que nuestros resultados, también los de otros autores se apartan de los argumentos anteriores, así el estudio de Branemark ${ }^{24}$ de 1969 muestra un alto porcentaje de éxito en ausencia de higiene y con inflamación en todos los pilares. Lekholm y cols ${ }^{5}$ encontraron correlación entre el índice de placa y el de gingivítis y la profundidad de bolsa periimplantaria, no obstante, afirman que un implante que tenga un aumento del sondaje no se acompaña necesariamente de cambios histológicos o microbiológicos que indiquen una periodontitis, resultados que concuerdan con los nuestros.

\section{CONCLUSIONES}

La aparición de recesión alrededor de un implante aumenta las probabilidades de fracaso del mismo.

Aunque existe una correlación positiva entre el índice de gingivitis, el de placa y la profundidad de bolsa, ninguno de los tres parámetros anteriores guarda relación con la pérdida de hueso ni con el fracaso del implante a los 2 años tras la conexión de la protesis a los implantes. Por tanto, estos parámetros nos indican la salud del surco gingival pero no las probabilidades de éxito del implante, ya que la inflamación inducida por la placa puede quedar restringida a la parte situada por encima del hueso.

\section{ABSTRACT}

The aim of this study was to determine, firstly, the relation between the failure of a sample of hydroxyapatite-coated implants (HA) and the periodontal parameters of the patient, and secondly, the degree of association between such parameters.

The sample was comprised of 52 patients and 158 H.A. coated implants (Integral ${ }^{\oplus}$ by Calcitek), which were revised three years after their insertion. Implant failure was determined according to the criteria of Schnitman and Shulman (1979).

A retrospective analytical observational follow-up study was carried out, by means of which the clinical and radiological evolution of the implants was determined. It was found that the peri-implant recession measured facial, lingual, mesial and distal from each implant is the only periodontal parameter associated with the failure of the implant $(\mathrm{p}=0.008)$. Bone resorption and periimplant recession were significantly associated $(p<0.05)$, as was probe depth with the gingivitis index in all positions and with the plaque index in the lingual position.

A positive correlation was found between the latter two indices $(r=0.519 ; p<0.01)$. It may be concluded that the appearance of recession around an implant increases the probability of its failure. There was also a positive correlation between the gingivitis index, the plaque index and pocket depth, which is indicative of the state of the gingival sulcus but not of the success rate of the implant.

\section{KEY WORDS}

Hydroxyapatite implant. Peri-implanted periodontal parameters. Osseointegration. Osseointegrated implant. Peri-implanted tissue. Peri-implantitis.

\section{BIBLIOGRAFÍA}

1. Lindhe J, Berlunch R, Ericsson I, Liljenberg B, Marinello C. Experimental Breakdown of peri-implant and periodon- 
tal tissues. A study in the beagle dog. Clin Oral implant Res 1992;3:9-16.

2. Brägger U, Bürgin W, Hämmerle CHF, Lang NP. Associations between clinical parameters assessed around implants and teeth. Clin Oral Imp Res 1997;8:412421.

3. Becker W, Becker BE, Newman MG \& Nyman S. Clinical and microbiological findings that may contribute to dental implant failure. Int $\mathrm{J}$ Oral Maxillofac implants 1990;5:31-38.

4. Nishimura K, Itoh T, Takaki K, Hosokaw R, Naito T, Yokota M. Periodontal parameters of osseointegrated dental implants. A 4-year controlled follow-up study. Clin Oral Impl Res 1997:8:272-278.

5. Lekholm U, Adell R, Branemark P-I, Eriksson B, Rockler B, Lindvall AM, Yoneyama T. Marginal tissue reactions at osseointegrated titanium fixtures. A cross-sectional retrospective study. Int $\mathrm{J}$ Oral Maxillofac Implants 1986;15:53-61.

6. Adell R. Lekholm U, Rockler B. Marginal tissue reactions at osseointegrated titatnium fixtures. (I) A 3 -Year longitudinal prospective study.Int J Oral Maxillofac Surg $1986 ; 15: 39-52$

7. Cox JF, Zarb GA. The longitudinal clinical efficacy of osseointegrated dental implants: a 3-year report. Int J Oral Maxillofac Implants 1987;2: 91-100.

8. Silness J, Löe H. Periodontal disease in pregnancy II. Correlation between oral hygiene and periodontal condition. Acta Odontol Scand 1953;2:153-62

9. Löe H. The Gingival Index, the Plaque Index, and the retención Index. J Periodontol 1967;38: 610-616.

10. Miller SC. Textbook of periodontia. 3rd ed. Philadelphia, $\mathrm{PA} ; 1950$.

11.Carranza FA, Newman MG. Periodontología clínica. $8^{\mathrm{a}}$ ed.Méjico:Mc Graw-Hill Interamericana;1998.

12. Rateitschak KH. Atlas de periodoncia. Barcelona: Salvat;1987.
13. Meijer HJA, Steen WHA, Bosman F. A comparison of methods to assess marginal bone height around endosseous implants. J Clin Periodontol 1993;20:250-53.

14. Schnitman PA, Shulman LB. Recommendations of the consensus development conference on dental implants J Am Dent Assoc 1979;98:373-77.

15. Ten Cate AR. The gingival junction. In:Branemark PI, Zarb GA, Albrektsson T,(eds). Tissue Integrated Prosthesis. Chicago: Quintessence Publishig Company 1985: 145153.

16. Misch CE. Implantología contemporánea. Madrid: Mosby/Doyma; 1995.

17. Lindhe J, Haffajee AD, Socransky SS. Progression of periodontal disease in the absense of periodontal therapy. J Clin Periodont 1983;10:433-42.

18 Nishimura K, Itoh T, Takaki K, Hosokaw R, Naito T, Yokota M. Periodontal parameters of osseointegrated dental implants. A 4-year controlled follow-up study. Clin Oral Impl Res 1997:8:272-78.

19. MC Kinney RV. Implantes dentales endoóseos. Londres: Mosby; 1993.

20. Ericsson I, Berglundh T, Marinello C, Liljenberg B, Lindhe J. Long-standing plaque and gingivitis at implants and teeth in the dog. Clin Oral Imp Res 1992;3:99-103.

21. Bert M. Les implants dentaires: bases fondamentales, techniques chirurgicales, applications cliniques. Paris: Ed CdP; 1987.

22. Leonhardt A, Berglundh T, Ericsson I, Dahlén G. Putative periodontal pathogens on titanium implants and teeeh in experimental gingivitis and periodontitis in dog. Clin. Oral Impl. Res 1992;3:112-19.

23. Steflik DE, Mc kinney R, Sisk A. Dental implants retrieved from humans: a diagnostic light microscopic review of the findings in seven cases of failure. Int J Oral Maxillofac Implants 1991;6: 147-53.

24. Branemark P, Breine U, Adell R, Hansson B. Intraosseous anchorage of dental prosthesis. I: experimental studies. Scand J Plast Reconstr Surg 1969;3:81-100. 
